\title{
GESTIÓN DEL TERCER SECTOR: UNA LUCHA COLECTIVA POR PERMANECER
}

\section{THIRD SECTOR MANAGEMENT: A COLLECTIVE STRUGGLE TO "STAY ALIVE"}

\author{
Jazmín Sánchez Manrique ${ }^{1}$, Valentina Schmitt ${ }^{2}$
}

\begin{abstract}
Palabras clave: Resumen
Tercer sector; $\quad$ La ayuda social es un tema que cada vez ha tomado mayor relevancia organizaciones no para la sociedad, por este motivo, la creación de organizaciones no gubernamentales; gubernamentales (ONG) ha sido de vital apoyo para hacer frente a la finanzas; gestión de recursos; voluntariado problemática que el Estado o sector privado no ha podido atender aún en su totalidad; sin embargo, su permanencia en el mercado se ha visto cuestionada por su limitado acceso a recursos económicos y no económicos. En ese sentido, el objetivo de esta investigación es determinar las variables importantes que permitan realizar una gestión íntegra.
\end{abstract}

\section{Códigos JEL: L30, L31}

Keywords:
Third sector;
non-governmental
organizations;
finance;
resource
management;
volunteering

\begin{abstract}
Social aid is an issue that has become increasingly important for society, for this reason, the creation of non-governmental organizations (NGOs) has been of vital support to be able to face the problems that the State or private sector has not. still able to attend in its entirety; however, its permanence in the market has been questioned due to its limited access to economic and non-economic resources. In this sense, the objective of this research is to determine the important variables that allow a complete management.
\end{abstract}

JEL Codes: L30, L31

\section{INTRODUCCIÓN}

Las dificultades de financiación de las ONG's son problemas que se han vuelto recurrentes, siendo uno de los principales motivos por los que pone en duda su permanencia, dada la dependencia que existe con las donaciones del sector privado y sociedad civil. La sostenibilidad y la captación de recursos de las Organizaciones No Gubernamentales (ONG) son temas que se encuentran relacionados directamente, siendo una de las más importantes para su desarrollo a lo largo del tiempo.

\footnotetext{
${ }^{1}$ Universidad de Piura, Facultad de Administración de Empresas, Calle Bellavista 199, Miraflores, Lima (Perú).

E-mail: jazminsanchezmanrique@gmail.com

${ }^{2}$ Universidad de Lima, Escuela de Posgrado, Facultad de Ciencias Empresariales y Económicas, Javier Prado Este 4600, Santiago de Surco, Lima (Perú).

E-mail: vschmitt@ulima.edu.pe
} 
Las ONG's, de acuerdo con el Alto Comisionado de las Naciones Unidas para los Refugiados son "Organizaciones independientes y sin ánimo de lucro que surgen a raíz de iniciativas civiles y populares y que por lo general están vinculadas a proyectos sociales, culturales, de desarrollo $\mathrm{u}$ otros que generen cambios estructurales en determinados espacios, comunidades, regiones o países" (ACNUR, 2017). Lograr que las ONG's sean sostenibles y generen impacto en la sociedad a la vez son algunos de los retos de sus gestores.

En el Perú, el Estado reconoce como ONG a las asociaciones, fundaciones y comités que se encuentran reguladas por el Código Civil. Al cierre del año 2017, según la Agencia Peruana de Cooperación Internacional (Durand, 2018), existían en el Perú aproximadamente 5000 ONG's, sin embargo, solo están obligadas a inscribirse en APCI aquellas que reciben cooperación directamente de gobiernos. De acuerdo con la base de datos de APCI, existen 1480 ONG's en el Perú, de las cuales están vigentes 715. Asimismo, nos indica la distribución de los sectores en los que se tiene intervención es de: $17 \%$ en educación, $16,3 \%$ en agricultura, $15 \%$ en temas de género y desarrollo social, $5,5 \%$ en trabajo y promoción social y $30 \%$ en temas diversos.

A su vez, cabe mencionar que las organizaciones tienen algunas dificultades en el tema de captación de recursos, debido a los siguientes factores: aumento de las ONG's a nivel nacional, tendencias cambiantes con respecto a las causas que se quiere apoyar con el pasar de los años, creación del sector privado de sus propias ONG's, entre otros. Si bien la sociedad civil se ha vuelto empática con estas causas, aún no tienen la disposición completa de aportar a través de recursos monetarios y no monetarios a este tipo de organizaciones, debido a que, no tienen conocimiento completo de cómo se ha empleado lo donado, teniendo así un alto grado de inseguridad.

Por ello, se ha investigado a una ONG a la cual se denominará Perú-1. Esta organización se encuentra ubicada en aproximadamente diecinueve países de América Latina y El Caribe.
Fue fundada en 1997 como una iniciativa de jóvenes que tenían como objetivo principal superar la situación de pobreza de los pobladores a través de la construcción de viviendas. Con el pasar del tiempo, esta iniciativa que se generó por un evento puntual empezó a replicarse en diferentes países, teniendo el día de hoy un alcance significativo y generando oportunidades de mejora a muchas familias de distintas comunidades. En el 2012, evaluaron su modelo de trabajo y observaron que el tema de hacinamiento no era la única necesidad; por este motivo, decidieron replantear su estrategia de aporte a la sociedad, determinando así, que también era necesario generar proyectos comunitarios, en pro del desarrollo de toda la comunidad.

Perú-1 tuvo como primera actividad en Perú la construcción de viviendas que se desarrolló luego del terremoto de Pisco en el 2007. Para el año 2019, se encuentra trabajando en aproximadamente cuatro distritos de Lima: Pachacámac, San Juan de Miraflores, San Juan de Lurigancho y Ventanilla; teniendo a su cargo a aproximadamente a veintiún comunidades y un aproximado de doscientos voluntarios como parte de equipo permanente de la organización. Asimismo, tiene como principales métodos de captación de recursos la colecta a nivel nacional (Lima, Trujillo y Piura) que se realiza una vez al año, donde los voluntarios toman las calles de la ciudad para recaudar dinero a través de latas. Adicionalmente, tienen las alianzas estratégicas con empresas privadas, las cuales se realizan por eventos específicos o convenios anuales. Estas actividades son variables a lo largo del año y al ellos no tener una certeza de los ingresos que se pueden generar, deja la planificación anual inestable.

El fin de desarrollar esta investigación fue encontrar una relación entre los métodos de captación de recursos y la sostenibilidad de una ONG; logrando así, determinar el factor que genera deficiencia en la relación de estas dos variables. Asimismo, recomendar buenas prácticas de otras organizaciones y que estas se adapten al modelo organizacional de Perú-1 buscando generar una fuente de ingresos directos para la organización, de tal modo que esta genere 
sostenibilidad a largo plazo y no dependa en su totalidad de la sociedad civil y empresas privadas.

\section{MARCO TEÓRICO}

\section{La gestión de ONG's}

La gestión de una ONG tiene como finalidad el optimizar los recursos disponibles, para garantizar el logro del fin social propuesto. Es fundamental tener en cuenta que el simple hecho de agrupar personas y recursos no es suficiente para garantizar la asignación de la mejor forma o la ejecución de las actividades correctas, para ello está la gestión (Tenório, 2006).

Los gestores son responsables ante los socios, fundadores y entidades; pero, sobre todo, al sector que atiende, dado que podrían verse desamparadas si la organización desapareciera. Asimismo, consideran que se debe realizar un diagnóstico para poder utilizar un instrumento eficaz que es la planificación estratégica, que se encarga de integrar el presente y el futuro creando condiciones que permitan gestionar oportunidades y evitar riesgos. Para desarrollarla se debe tener en cuenta tres factores: la situación de la organización, metas planificadas y acciones para lograr las metas (Asís, Gross, Lillo, \& Caro, 2003).

Para desarrollar un excelente sistema de gestión se debe considerar tres pilares fundamentales: Gestión de ética, detallando las normas y códigos éticos; normas y sellos referidos a gestión de la igualdad, ambiente y seguridad; y, por último, la gestión de calidad, donde será un proceso de mejora continua que no solo implica la modificación de estrategia sino un cambio cultural de la gestión de la organización (Barbero, 2008). Esos factores permiten que todos los miembros de la organización sean partícipes de ello buscando generar un valor añadido para el producto o servicio.

Dentro de la gestión de las organizaciones sin fines de lucro, existe un factor del mismo nivel de importancia que los recursos económicos y es el capital humano que se ve reflejado en los voluntarios. Por este motivo, se debe tener en cuenta que el voluntario y la organización generan un mutuo acuerdo, donde ambas partes entregan acciones de valor. Por el lado de la organización, se debe comprometer a entregar objetivos por los cuales trabajar, instrumentos que le permitan realizar la labor encomendada. A su vez, el voluntario entregará a cambio su ilusión, tiempo y capacidad de servicio. Aparentemente, este intercambio no tiene un coste. Sin embargo, la organización debe ser consciente de la necesidad de planificar recursos económicos y tiempo necesario exclusivamente para la gestión de los voluntarios, lo cual inicia en el proceso de selección e incorporación de los voluntarios, luego da paso a la fase de desarrollo y retención, para finalizar evaluación, seguimiento, control y salida de los voluntarios (Sajardo \& Sierra, 2008).

La sociedad civil y el tercer sector son ámbitos sumamente relacionados. El segundo es una consecuencia de la unión de un conjunto de personas de la sociedad civil, que tienen un grado de frustración por la falta de expresión económica y política que encuentran en muchos lugares del entorno donde viven. Esto se debe a la duda que tienen con respecto a la capacidad del Estado para hacer frente a problemas de desarrollo, bienestar social y medio ambiente (Lester, Helmunt, List, Toepler, \& Wojciech, 1999).

Asimismo, el tercer sector tiene un carácter privado, a fin de satisfacer las necesidades colectivas y de interés público buscando así poder redefinir las formas de relacionarse entre la empresa privada, el Estado y la sociedad civil; creando así una mayor integración moral entre los ciudadanos (Portocarrero, Sanborn, Cueva, \& Millán, 2002).

Si bien las ONG's no son entidades que busquen obtener beneficios sino generar un impacto, terminan acercando su gestión a la de las empresas, dado que, brindan algún producto o servicio con el fin de tener una fuente adicional de ingreso. Por este motivo, es relevante el poder trabajar el posicionamiento de ellos como organización y marca. Una variable importante es 
la percepción, dado que, es aquel significado que se basa en las experiencias atribuyéndose a los estímulos que entran por los sentidos (Costa, 1992).

Adicionalmente, garantizar un adecuado posicionamiento es el paso más importante en una venta efectiva (Ries \& Trout, 1989). Lo mencionado responde a que la sociedad civil puede aportar a organizaciones del tercer sector que se encuentren en el país donde radica o en el exterior y es ahí donde el cliente debe tener una percepción clara cuál es la misión de la organización y si se siente identificado con está llegando así a una fidelización que debe mantener a lo largo del tiempo. Por este motivo, se deben tener en cuenta ciertos elementos para la adecuada fidelización: un marketing relacional adecuado, política del cliente objetivo y una gestión del valor percibido (Apaolaza, Forcada, \& Hartmann, 2002).

Las organizaciones sin fines de lucro para poder sostenerse en el tiempo reciben recursos económicos y no económicos de entidades privadas y públicas sin contraprestaciones, teniendo así un régimen fiscal favorable. Por este motivo, es necesario que se rindan cuentas en función a los recursos recibidos con el fin de demostrar que se están usando de modo adecuado y para el fin solicitado. Adicionalmente, esta información permite a los donantes y sociedad civil conocer las actividades realizadas por la organización, los medios y recursos que tiene, situación económica-financiera, nivel de cumplimiento de presupuestos y resultados y la capacidad de continuar prestando servicios en el futuro (Fuentes, 2007).

La rendición de cuentas en términos contables puede resultar muy favorecedor para las ONG's logrando que se conviertan en eficaces para el logro de la implementación de los objetivos planeados (Bonbright, 2007). Dentro de las principales actividades que deben realizar para que se dé una efectiva rendición de cuentas está el diálogo con los participantes directos e indirectos que existen en el entorno y quienes se llevan una imagen de la organización. Se debe brindar informes públicos de los estados financieros, actividades que se llevaron a cabo, entre otros, pero se debe hacer un especial énfasis en el resultado que se obtuvo y cuál fue el impacto que se generó en la sociedad elegida.

Una correcta comunicación, afirma Bonbright (2007), permite que se conozca qué es lo que se hace como organización lo cual la lleva a ganar un prestigio. Aún, el autor determina tres variables importantes para que las ONG's sean efectivas cuando involucran a la persona del entorno:

1. Escuchar a los constituyentes y demostrar su impacto;

2. la respuesta convencional comparará los indicadores que no llegan a describir el impacto social;

3. la comunicación a los terceros sobre el trabajo de manera efectiva.

Sin embargo, se debe tener en consideración que en muchas ocasiones la medición del rendimiento de estas organizaciones sigue siendo un aspecto sin resolver, dado que el sistema resultante de los cálculos efectuados nunca proporcionará una base correctamente adecuada para planificar o medir el desempeño como lo hace la medida del beneficio de las organizaciones lucrativas (Álvarez, Vásquez, \& Santos).

Esto se debe principalmente a que las entidades no lucrativas tienen como función brindar servicios a la sociedad que son en su mayoría gratuitos o a un precio mucho más bajo que el costo, por lo que la gestión de las organizaciones se verá orientada a brindar la mayor cantidad de servicios con los recursos disponibles y el éxito va a depender del grado en que se consiga (Fernández \& Pablos, 1996).

Por ello, la rendición de cuentas debe abarcar por lo menos cinco áreas (Vernis, 2001):

1. Área financiera/fiscal, se deberá explicar con detalle el uso que se hace con respecto 
a los aportes recibidos y el cumplimiento de sus obligaciones legales;

2. Área de gestión, demostrar la utilización de procedimientos adecuados de gestión, con criterios de eficiencia y eficacia;

3. Área de actividades, rendir cuentas de cómo realiza actividades y proyectos con el fin de demostrar que los proyectos de implementan buscando un alto nivel de calidad maximizando los beneficios de los usuarios;

4. Área directiva, descripción de las personas responsables de dirigir la organización;

5. Área estratégica, demostrar que la organización enfoca su trabajo al cumplimiento de la misión y aportando el mayor valor añadido a la sociedad.

Finalmente, para poder tener una sostenibilidad dentro de la organización, no solo debe estar conformado por los correctos resultados económicos, sino que se debe generar una buena relación con los grupos de interés buscando rentabilidad social y medioambiental (Pradini \& Sánchez, 2007). Por este motivo, deben generar el empoderamiento de las personas a las que se dirigen siendo este su auténtica misión y verdadero valor añadido que les permitirá ser sostenibles y efectivos en sus acciones (Balas, 2008).

Teniendo así un resultado en función al impacto social de los proyectos desarrollados por la organización, nivel de cumplimiento de su misión, efecto sobre los beneficiarios, entre otros (Fuentes, 2007).

\section{La captación de recursos en las ONG's}

La captación de fondos es una actividad orientada a obtener fondos públicos o privados para financiar las actividades de las ONG's. Sin embargo, se debería usar el termino de captación de recursos, dado que, este tipo de organizaciones no solo busca captar recursos económicos, sino también otro tipo de recursos como bienes o servicios. Entre las formas de captación de recursos para ONG's pueden estar los beneficios de orden tributario (como inmunidades $y$ exenciones), las subvenciones, los convenios y alianzas y, dependiendo de la legislación del país, las donaciones deducibles de impuestos (Barbosa \& Oliviera, 2007).

Dentro de equipo que se debe considerar para una adecuada captación de recursos son personas con conocimientos en marketing, ventas, comunicación y finanzas. Existen muchas formas de recaudar recursos, la selección de cada uno de ellos dependerá de los recursos económicos o conocimientos técnicos que requieran. Por este motivo existen criterios de elección de la forma en la cual se captarán los recursos (Pérez, 2006):

- La popularidad de la causa que abandera la organización: si tiene una causa que no es muy conocida o no está dentro de las prioridades de apoyo de las personas, tendrá dificultad de encontrar apoyo.

- Cantidad o tipo de recursos que exigen: puede una forma de captar recursos ser muy adecuada para la organización; sin embargo, esta puede carecer tanto de recursos económicos como de voluntarios para la ejecución de este.

- Técnicas que vayan en relación con la misión, valores y cultura interna de la organización: permite orientar que tipo de técnica es la adecuada para cada organización.

Dentro de los métodos más comunes se tienen los siguientes (Pérez, 2006):

- Peticiones cara a cara: Consiste en solicitar donación o afiliación a un individuo o empresa siendo una de las formas más sencillas de recaudar fondos siempre y cuando se realice en pequeñas escalas. La petición de puede realizar a través de cartas o llamadas telefónicas; sin embargo, implica mucho tiempo de preparación y ejecución.

- Captación de socios en la calle: Método eficaz para organizaciones notorias o 
aquellas que abanderen una causa que sea identificada por la sociedad. Asimismo, para hacer un socio se tiene que abordar a cien personas con conversaciones aproximadas de dos minutos.

- Colectas: Forma de solicitud a escala personal a escala masiva; se pueden dar desde sencillas operaciones de recojo de donativos entre los asistentes a un evento o eventos de escala nacional.

- Anuncios e información en medios de comunicación: Realizar publicidad a través de anuncios de prensa, radio, televisión y otros medios los cuales le permiten dar a conocer su causa de forma masiva, que funcionará de manera más efectiva si se combina con acciones de relaciones públicas adecuadas. Sin embargo, suele ser una de las formas con costo más elevado.

- Correo directo: Envío de una solicitud de apoyo económico a través de un correo electrónico. Para considerar a la lista de personas que se desea enviar, se debe tener en consideración si el perfil del público corresponde con el de las personas que se desea captar, frecuencia con la cual se actualiza la lista con el fin de eliminar direcciones anticuadas y margen de error de $-5 \%$ de direcciones invalidas.

- Solicitudes por teléfono: Método costoso pero eficaz, su rentabilidad se basará si se usa con personas que ya generaron un vínculo con la organización con el fin de fidelizarlas. La principal ventaja es el permitir un diálogo personal con el interlocutor, por lo que pueden hacer frente a las dudas que se presenten $\mathrm{y}$ consolidar la acción de manera rápida; la desventaja es el no poder explayarse.

- Ciberfundraising: Captación de fondos mediante el uso de internet; asimismo, el donante online se involucra fácilmente a través de acciones en la red. Dentro de las principales ventajas se encuentra la cobertura, eficiencia, rapidez, flexibilidad y permanencia.

- Apoyo de organizaciones no lucrativas: Entidades que se suponen al apoyo no necesariamente a través de recursos económicos, sino también a través de bienes y servicios.

- Venta de artículos: Venta de material promocional como polos, llaveros, tazas, lapiceros, entre otros. Permite ofrecer a las personas la oportunidad de mostrar su apoyo a la causa y así permite poder generar promoción a la organización.

- Eventos: reuniones pequeñas, cenas benéficas, concierto, exposiciones, rifas, mercadillos, ciclos de cine, entre otros. La mayoría de las veces ello conlleva un riesgo, razón por la cual se debe realizar de manera cuidadosa en cada una de las etapas con el fin de reducir la probabilidad de fracaso.

- Colaboración de empresas: Se tienen diversas opciones de realizar el trabajo en conjunto como solicitud de empresas de un porcentaje de sus ingresos, colectas entre los empleados, promociones conjuntas. Ello dependerá de la capacidad de poder generar confianza, compromiso y una clara gestión; para poder llevarlo a cabo se deberá realizar convenios o acuerdos escritos donde se manifiesten los objetivos, resultados, responsables, entre otros (Gavilán, Iglesias, \& Beitia, 2013).

El emprendedurismo social presta una eminente contribución en la profesionalización de la gestión en el ámbito del Tercer Sector, que debe ser aliada a la cultura de la sistematización de las actividades, así como del acompañamiento de las tendencias sobre las mejores prácticas de gestión efectiva (Alves, Faria, \& Fontenele, 2009). Es por este motivo que, se debe buscar proporcionar el contenido correcto a las personas adecuadas y en el momento adecuado para que tomen mejores decisiones (Cordobés, Sureda, \& Carreras, 2007). 


\section{METODOLOGÍA}

Para fines de esta investigación se tuvo como referencia el estudio de caso de una ONG de Perú. Asimismo, se empleó el método analítico sintético que se define como el estudio de situaciones, descomponiéndolo en partes de tal modo que pueda analizar cada una de ellas, para luego buscar la integración y estudiarla como un todo; dado que, es una investigación que tiene variables cualitativas y cuantitativas (Bernal, 2010).

El tipo de estudio es descriptivo y explicativo, debido a que, se busca determinar si existe relación entre las variables planteadas (los distintos tipos de recaudación de fondos) y la sostenibilidad de la organización. A su vez, se realizó la evaluación documental, al tener acceso a documentos financieros de la organización en evaluación, así como los reportes que ellos generaban mensualmente desde el año 2015 hasta el año 2018.

La investigación buscó profundizar en los conocimientos establecidos sobre recaudación de fondos (investigación básica), para poder llevar a cabo la búsqueda de una solución efectiva con respecto a la sostenibilidad de la organización (investigación aplicada). Por tanto, en la recolección de datos se trabajó con encuestas y entrevistas. Para poder determinar la cantidad de muestra de encuestas se tomó como referencia muestreo para poblaciones finitas, dando como resultado una muestra de 57 personas. Agregado a ello, se realizaron dos entrevistas a profundidad con el fin de poder conocer la situación desde la perspectiva de los directores de la ONG. Después de analizados los datos cualitativos y cuantitativos, se utilizó de la triangulación de datos para llegar a discusión de resultados y presentación de las conclusiones.

\section{RESULTADOS}

La gestión de la organización está a cargo del área comercial liderada por el Gerente General, quien tiene a su cargo la dirección de desarrollo de fondos, comunicaciones y finanzas, quienes se encargan de poder gestionar las propuestas para ser presentadas a las entidades correspondientes.
Asimismo, trabaja de manera muy cercana con la Dirección Social quienes se encargan de obtener información sobre las necesidades y generar proyectos para las comunidades en las cuales tiene intervención activa.

Han intentado a través de diferentes métodos lograr captar recursos tales como: colectas anuales, alianzas anuales (voluntariado corporativo), proyectos comunitarios, construcción de viviendas, donación de vueltos, conciertos, aporte de fondos y organismos internacionales, entre otros. Sin embargo, ninguna de estas es sostenible en el tiempo. Un ejemplo es que, con respecto a la colecta nacional realizada en el año 2017, solo una provincia logró cumplir la meta y a nivel de Lima Metropolitana solo dos distritos. A nivel general solo se logró cumplir el $52.24 \%$ de la meta. Las consecuencias de estos incumplimientos son no poder cumplir con los costos fijos; el logro de cumplir con la meta de la colecta implica un $15 \%$ en la cobertura de costos fijos de manera anual.

Uno de los productos con los que ingresó la organización al mercado fue a través de la construcción de viviendas; sin embargo, esto no ha sido creciente con el pasar de los años. La construcción de viviendas de emergencia implica un costo aproximado de S/ 5000 por cada una de ellas, lo cual, para una ONG es un costo alto. Esto se debe principalmente a la madera, dado que, se usa pino con el fin de que a vivienda dure en el tiempo bajo los cuidados específicos.

Para el año 2018, la construcción de viviendas no se estuvo dentro de los principales temas de ayuda social; si bien el tema de hacinamiento debería tenerse en cuenta, no es un producto atractivo para las empresas como venta de voluntariado corporativo por el alto costo que implica y la poca cantidad de voluntarios que se puede tener por casa (un aproximado de ocho). En promedio, se construye un aproximado de 139 viviendas (este número no incluye el año 2017 por ser un año atípico por la Fenómeno del Niño), de las cuales el $50 \%$ es netamente financiado por la organización y la diferencia por la venta de voluntariado corporativo. 
Además, un factor que se debe tener en cuenta para este producto emblema es la coyuntura ambiental que se vive; la madera es un producto que contribuye a la tala de árboles, servicio que a la fecha es muy cuestionado por la alta deforestación que se tiene en el planeta.

Dada la coyuntura cambiante, la organización ha tenido que hacer frente con la implementación de nuevos productos como es el desarrollo de proyectos comunitarios. Ello busca que las empresas privadas financien diferentes necesidades que tienen las comunidades como son el muro de contención, implementación parques, creación de bibliotecas, arborización, entre otros. Dentro de las principales ventajas se encuentra que puedes movilizar a una mayor cantidad de voluntarios corporativos, el costo de ejecución es mucho más bajo que construir una vivienda y el impacto social es mayor; sin embargo, dentro de las deficiencias que tienen, es el no tener al personal especializado para la planificación e implementación de ello.

En función al año 2017, han surgido mejoras en los procesos de calidad, sin embargo, se ha dado con ratios bajos en eficacia. Dentro de los principales retos que tienen para poder mantener este producto en su cartera y ofrecerlo a las empresas está en mejorar el ratio de comunidades activas con mesas de trabajo y tener un cumplimiento mínimo del $95 \%$ de planes de acción en las mesas de trabajo correspondientes; aumentar el número de coordinadores de comunidad y la captación de estos, por el proceso de apertura de nuevas comunidades.

En 2018, la organización tuvo en un momento de equilibrio, en el primer semestre hubo un cumplimiento del $79 \%$ con respecto a lo planificado con respecto a los ingresos. Mensualmente, ha logrado cumplir la meta de ingresos; sin embargo, no se debe tener en consideración los meses de enero y febrero, dado que, esos ingresos eran de contratos con empresas del año 2017 que, por problemas de sistemas, no ingresó en el mes correspondiente. El sector del cual ellos dependen como organización es el privado, aún no han logrado establecer estrategias efectivas para poder crear un vínculo con la sociedad civil a pesar de las diversas propuestas que han podido generar.

Para lograr que la sociedad civil sea el principal donante, tienen planificado realizar inversiones en estudios de posicionamiento, puesto que, a través de ello podrá determinar la forma adecuada en la cual debe hacer su planificación para llegar a su público objetivo. Asimismo, el 2018, tuvo como foco principal, el producto de socios; este se basa en el monto debitado mensualmente de las tarjetas de crédito o débito de las personas que deciden aceptar. Desde el 2012, la recaudación del plan de socios ha sido constante de manera anual, sin tener un plan para la captación de estos.

Con respecto a los egresos, a nivel semestral han tenido un incumplimiento del $4 \%$ para el primer semestre del 2018, el cual no es significativo. Sin embargo, se debe considerar el segundo semestre, dado que, si no se tiene un back-up correcto puede ser el inicio de incumplimientos de pago tanto a su personal contratado como a los proveedores. La mayor parte de egresos es por costos variables, los cuales implican, el desarrollo de los proyectos comunitarios y actividades que no necesariamente son planificadas. Sin embargo, el costo fijo no se encuentra tan alejado, una de las medidas tomadas fue cambiar de lugar físico de la oficina para poder disminuir ello.

Por otro lado, las ONG's tienen la fuerza principal de trabajo en los voluntarios, para lo cual, se tiene un ciclo del voluntario el cual empieza con el proceso de inserción y concluye en el proceso de salida; sin embargo, ello no necesariamente se aplica a la realidad. Existen deficiencias al momento en el cual el voluntario pasa a ser parte de la organización, dado que, no hay un plan específico de desarrollo. Con eso, carecen de un plan en el cual velen que el voluntario tenga roles y responsabilidades que cumplir en actividades dentro de la organización, con lo cual, no logran que la persona que apropie de la filosofía que tienen; todo ello ha traído como consecuencia que el tiempo de permanencia de las personas en el equipo permanente sea de tres meses en promedio, donde esto dificulta poder generar planes a largo plazo y continuidad de los 
proyectos. Adicionalmente, el equipo de contratados, también se encuentra en un proceso de cambios, buscando encontrar una estructura adecuada que responda a la necesidad de la organización, así como a la demanda que tienen por parte del sector privado.

Agregado a ello, de acuerdo con la muestra, se puede afirmar que solo el $35 \%$ de ellos conoce en qué se invierte los fondos recaudados, es importante, dado que el ser humano actúa en función a experiencias y si este no tiene referencia de qué es lo que se hace específicamente con ese dinero, en muchos casos, tiende a no repetir la acción. Asimismo, la organización no está teniendo una gestión que responda a la necesidad de sus voluntarios, lo cual se puede ver reflejado en la alta rotación que tienen estos; no se ha establecido una correcta estrategia de fidelización y si no se tiene fidelizado a este cliente interno no se podrá ver reflejada la identidad de la organización ante a la sociedad. A pesar de que cuentan con un visor sobre temas financieros, este no es de conocimiento de todo el público interesado.

Asimismo, el $42,5 \%$ en promedio de los encuestados tiene conocimiento de las actividades que realiza la organización con el dinero que se ha recaudado a través de los diferentes canales. Estas personas que tienen este conocimiento son voluntarios que llevan más de cinco años dentro de la organización, con lo cual, el grado de conocimiento está en función a la cantidad de años que uno está en la organización. Se debe tener en consideración, que esta cantidad de voluntarios con gran experiencia dentro de la organización debe ser referente en la consideración de implementación de proyectos de captación de recursos, dado que, muchos de ellos trabajan en el sector privado, siendo ellos una de las principales fuentes de apoyo que se debe tener para la implementación de nuevas estrategias.

Adicionalmente, de la entrevista realizada a los gestores de la organización, se puede observar que si bien se conoce que la situación de la institución no es algo reciente. No se han tomado medidas que hayan sido efectivas dada la carencia de conocimiento específico en el tema de captación de recursos y marketing. A su vez, han sufrido un retraso en la adaptación del entorno, con lo cual, se han visto afectados de manera directa. Otro problema que se puede identificar es que no se ha generado una estrategia de marketing específica para la retención de las empresas con las que han venido trabajando a lo largo de los años lo cual pone en peligro la relación y durabilidad de las alianzas anuales fuente del $50 \%$ de sus ingresos. No tienen conocimiento del por qué dejaron de trabajar con ellos.

\section{CONCLUSIONES}

Esta organización ha logrado un posicionamiento y reconocimiento en el mercado, lo cual le ha permitido generar la confianza de voluntarios, empresas privadas y sociedad civil. Generando así alianzas anuales con empresas importantes, nacionales e internacionales, y un promedio de 198 voluntarios en el año. Ese apoyo, como parte del equipo permanente genera soporte al desarrollo de las actividades como construcción de viviendas, proyectos comunitarios, relevamiento de información, entre otros.

La relación que la organización ha podido generar con las distintas comunidades es un factor importante. Eso les ha permitido conocer y gestionar proyectos relacionados a sus comunidades. Asimismo, el desarrollo de estas no solo está enfocada en temas de infraestructura como renovación de áreas verdes e implementación de bibliotecas, sino que también ha permitido que los líderes comunitarios desarrollen habilidades que les permitan desenvolverse adecuadamente en el campo profesional y personal.

Sin embargo, a pesar de tener estos factores a su favor, existen otros que les han generado dificultades para el desarrollo de sus actividades. Uno de ellos es la autonomía para la toma de decisiones en función a inversiones. Eso se debe a que es indispensable que toda la junta directiva de la organización (compuesta por representantes externos y la gerente general) estén de acuerdo con las inversiones de montos elevados. Es así como, esta decisión depende en su mayoría del apetito de riesgo que pueda adquirir tanto la organización como las personas responsables de esta toma de decisiones. 
En definitiva, el pequeño grado de riesgo que desean asumir se debe principalmente a que los gestores no tienen el conocimiento adecuado de cómo gestionar los recursos económicos de tal modo que les permitan ser sostenibles en el tiempo. Si bien la experiencia de la organización se centra en el desarrollo de comunidades, en temas de sostenibilidad y rentabilidad aún se encuentran en estado de aprendizaje. Pero dada la coyuntura donde se crean organizaciones que brindan apoyo a la sociedad de manera tan frecuente, se debe solicitar recursos externos que les permitan buscar la manera óptima y alineada a la organización de generar recursos económicos.

Por otro lado, para llevar a cabo una adecuada estrategia de recaudación de fondos es importante poder implementar un equipo de trabajo que se encargue de realizar una investigación de campo. De esta manera se tendría oportunidades de investigar diferentes tipos de recaudación de fondos, que permitan adaptar al contexto en el cual se encuentra.

Finalmente, la retención de voluntarios es una variable la cual siguen intentando poder encontrar un modelo que les permitan generar una retención mayor a tres meses; dado que, en ese corto tiempo no se puede generar un trabajo continuo. Adicionalmente, a pesar de contar con una línea de proceso de estadía del voluntario, no se tienen definidos cuál es el flujo de roles que debería asumir con el fin de poder desarrollar habilidades que le permitan desenvolverse en el plano personal y profesional, siendo consciente de lo que sucede en su país. Dentro de las recomendaciones que se tienen para la organización es poder establecer un plan de entrada, seguimiento y salida de voluntarios, ello con el fin de poder aprovechar los conocimientos y el aporte que puedan generar en la organización, así como el brindarles la mejor experiencia y que conozcan la otra realidad del Perú.

\section{REFERENCIAS}

ACNUR, A. C. (enero de 2017). ACNUR. Obtenido de https://eacnur.org/blog/una-ongfuncion-social-

tc_alt45664n_o_pstn_o_pst/\#: :text=Las\%20ON
G\%20son\%20organizaciones\%20independientes ,\%2C $\% 20$ comunidades $\% 2 \mathrm{C} \% 20$ regiones $\% 20 \mathrm{o} \%$ 20pa\%C3\%ADses.

Álvarez, L., Vásquez, R., \& Santos, M. (s.f.). Resultados de la orientación al mercado: Evidencias empíricas de las organizaciones no lucrativas. Investigaciones Europeas de Dirección y Economía de la Empresa, 5(3), 1940.

Alves, M., Faria, M., \& Fontenele, R. (2009). Gestão nas Organizações do Terceiro Setor: Contribuição para um Novo Paradigma nos Empreendimentos Sociais. XXXVIII Encontro da ANPAD. Obtenido de http://www.anpad.org.br/admin/pdf/EOR1546.pd $\mathrm{f}$

Apaolaza, V., Forcada, F., \& Hartmann, P. (2002). El efecto del posicionamiento en la lealtad del cliente: Un análisis empírico para el caso de Iberdrola. Obtenido de http://www.ehu.eus/cuadernosdegestion/docume ntos/225.pdf

Asís, A. D., Gross, D., Lillo, E., \& Caro, A. (2003). Manual de Ayuda para la Gestión de Entidades No Lucrativas. Madrid: Fundación Luis Vives. Obtenido de https://www.pluralismoyconvivencia.es/upload/2 6/75/Manual_gestion.pdf

Balas, M. (2008). Organizaciones No Gubernamentales. Revista Española del Tercer Sector, 17-37.

Barbero, I. (2008). Sistemas de Gestión de la Calidad en el Tercer Sector de Acción Social: Situación Actual y Propuestas de Futuro. Obtenido de www.plan\%20de\%20tesis\%201/PT2/DialnetSistemasDeGestionDeLaCalidadEnElTercerSect orDeAcci-2695564\%20(3).pdf

Barbosa, M., \& Oliviera, F. (2007). Manual de ONGs: guia prático de orientação jurídica. Río de Janeiro: FGV.

Bernal, C. (2010). Metodología de la Investigación. Bogotá: Pearson.

Bonbright, D. (2007). El rostro cambiante de la rendición de cuentas de las ONG. Obtenido de 
http://rendircuentas.org/wp-

content/uploads/2010/10/El-rostro- cambiantede-la-rendicion-de-cuentas-de-las-ONG-David-

Bonbright.pdf

Cordobés, M., Sureda, M., \& Carreras, I. (2007).

ONG del conocimiento: Influir para el impacto social.

Obtenido

de

https://www.pwc.es/es/publicaciones/economia-

social/assets/ong-conocimiento-pwc.pdf

Costa, J. D. (1992). Diccionario de Mercado y

Publicidad. Caracas: Panapo.

Durand, P. (2018). Regulación sobre Supervisión de Actividades y Financiamiento de los Organismos No Gubernamentales en Perú, España y Chile. Investigación, Congreso de la República del Perú, Departamente de Investigación y Documentación Parlamentaria, Lima. Obtenido de http://www2.congreso.gob.pe/sicr/cendocbib/con 5_uibd.nsf/994910FB432068F20525833F00634 CBF/\$FILE/REGULACI\%C3\%93N_SOBRE_S UPERVISI\%C3\%93N_DE_ACTIVIDADES_Y _FINANCIAMIENTO_N_19.pdf

Fernández, J., \& Pablos, J. (1996). El proceso de gestión de las entidades no lucrativas y las normas de información presupuestaria elaborada por el Instituto de Contabilidad y Auditoría de Cuentas (ICAC). Actualidad Financiera, Monográfico Contabilidad, 51-65.

Fuentes, J. (2007). Las organizaciones lucrativas: Necesidades de los usuarios de la información financiera. Obtenido de https://dialnet.unirioja.es/servlet/articulo?codigo $=2376721$

Gavilán, B., Iglesias, J., \& Beitia, P. (2013). La captación de recursos del tercer sector. Obtenido de

http://www.3sbizkaia.org/Archivos/Documentos/ Enlaces/1561_3sgestion08.pdf

Lester, S., Helmunt, A., List, R., Toepler, S., \& Wojciech, S. (1999). La Sociedad Civil Global:
Las dimensiones del sector no lucrativo. Obtenido de

http://www.grupobbva.com/TLFU/dat/lasocieda dcivilglobal.pdf

Pérez, A. (2006). Introducción a la captación de fondos. Obtenido de https://s3.amazonaws.com/academia.edu.docume nts/34213947/captacion_fondos.pdf?AWSAcces sKeyId=AKIAIWOWYYGZ2Y53UL3A\&Expir es $=1555455761 \&$ Signature $=876$ Do97 $\mathrm{cDp} \% 2 \mathrm{Fn}$ xN6fGBuhUvnEcMk\%3D\&response-contentdisposition=inline $\% 3 \mathrm{~B} \% 20$ filename $\% 3$ DIntrodu ccion_a_la_captacio

Portocarrero, F., Sanborn, C., Cueva, H., \& Millán, A. (2002). Más allá del individualismo: El tercer sector en el Perú. Obtenido de https://books.google.com.pe/books/about/M\%C3 \%A1s_all\%C3\%A1_del_individualismo.h tml?id=8IJHv34Igx0C\&printsec $=$ frontcover\&so urce $=\mathrm{kp} \_$read_button\&redir_esc $=\mathrm{y} \# \mathrm{v}=\mathrm{one}$ page $\& \mathrm{q} \& \mathrm{f}=$ false

Pradini, J., \& Sánchez, E. (2007). La responsabilidad social en el tercer sector salud y drogas. Obtenido de https://dialnet.unirioja.es/servlet/articulo?codigo $=2327545$

Ries, A., \& Trout, J. (1989). Posicionamiento. Madrid.

Sajardo, A., \& Sierra, I. (2008). Avances recientes en la investigación económica sobre el voluntariado: valoración económica del trabajo voluntario, costes de gestión del voluntariado y voluntariado corporativo. Obtenido de http://www.redalyc.org/pdf/174/17412307008.pd f

Tenório, F. (2006). Gestão de ONGs: principais funções gerenciais. Río de Janeiro: FGV.

Vernis, A. (2001). Los diferentes elementos de la rendición de cuentas en las organizaciones no lucrativas. Madrid: Fundación Lealtad. 